\title{
Análisis de falla en la chapa de acero al silicio en transformadores de distribución. Efecto de la zona de precedencia de los transformadores
}

\section{Failure analysis in silicon steel sheet in distribution transformers. Effect of the zone of precedence of the transformers}

\section{Análise de falhas em chapa de aço silício em transformadores de distribuição. Efeito transformers área precedência}

\author{
María Gabriela Mago ${ }^{*} ;$ Luis Valles$^{2} ;$ Jhon Jairo Olaya ${ }^{3}$; Edgar Espejo ${ }^{3} ;$ PedroArango ${ }^{4} ;$ Mauricio Sierra $^{1}$ \\ 1Programa de Ingeniería Mecánica, Facultad de Ingeniería. Universidad Libre. Bogotá, Colombia \\ ${ }^{2}$ Centro de Confiabilidad, Área de Estudios de Postgrado, Facultad de Ingeniería, Universidad de Carabobo, \\ Valencia, Venezuela. \\ ${ }^{3}$ Centro de Materiales y Procesos, Facultad de Ingeniería, Universidad Nacional de Colombia. Bogotá, Colombia.. \\ 4. Laboratorio de Física del Plasma. Facultad de Ciencias Exactas y Naturales, Universidad Nacional de Colombia, \\ Manizales, Colombia. \\ *mariag.magor@unilibrebog.edu.co
}

Fecha Recepción: 6 de marzo de 2017

Fecha Aceptación: 30 de julio de 2017

\begin{abstract}
Resumen
El análisis de falla en la chapa de acero al silicio en transformadores de distribución presenta un diagnóstico basado en la micro estructura y propiedades magnéticas de este material, con el cual se fabrica el núcleo de los transformadores de distribución monofásicos de poste. Tales diagnósticos, complementan estudios realizados para evaluar la vida útil de estos equipos, comparando la calidad de los materiales con los que son fabricados aplicando "métodos no convencionales", cuando presentan condiciones de fallas, incrementando la experticia en los sistemas de distribución de energía eléctrica. El trabajo fue realizado aplicando técnicas de caracterización a la chapa de acero utilizando difracción de rayos $X$ o XRD y microscopía electrónica de barrido en ambiente o E-SEM, lo que permitió una visión más clara de los fenómenos que intervienen en el comportamiento de la chapa de acero de silicio. Aunque previamente se había realizado análisis metalográfico a muestras de equipos fallados para verificar el tamaño de grano, impurezas o inclusiones que hubieran podido afectar a la calidad del material durante su funcionamiento, se aplica XRD y se utiliza la ecuación de Scherrer comprobando el tamaño de los cristales (granos) en escala nanométrica, y luego, con la fórmula de Steinmetz se evalúan las pérdidas debido a la histéresis magnética que también afecta de manera importante el funcionamiento del equipo. Las Pérdidas Totales del Núcleo se deben a presencia de óxidos Tipo D y cambios en el tamaño del cristal (grano)
\end{abstract}

Palabras clave: Análisis de fallas, chapa de acero al silicio, transformadores de distribución, XRD y E-SEM.

\section{Abstract}

Failure analysis in the silicon steel sheet in distribution transformers presents a model based on the microstructure and magnetic properties of this material diagnosis, with which the core of the single-phase distribution transformers pole is manufactured. Such diagnoses, complementary studies to evaluate the life of these teams, comparing the quality of the materials that are manufactured using "unconventional methods" when present fault conditions, increasing expertise in distribution systems of electric power. The work was performed using techniques of characterization to the steel sheet using (X-ray diffraction) 
or XRD and SEM in environment (scanning electron microscopy) or E-SEM allowing a clearer view of the phenomena involved the behavior of the silicon steel sheet. Although previously performed metallographic samples of failed to check the size of grain impurities or inclusions that might have affected the quality of the material during operation equipment analysis, XRD is applied and the Scherrer equation is used by checking the size of the crystals (grains) in the nanoscale, and then with the formula Steinmetz losses are evaluated due to magnetic hysteresis also affects significantly the operation. Total Core Losses are due to the presence of Type D oxides and changes in crystal size (grain)

Keywords: Failure analysis, silicon steel sheet, distribution transformers, XRD and SEM-E.

\section{Resumo}

Análise de falha na chapa de aço silício em transformadores de distribuição apresenta um modelo baseado na microestrutura e propriedades magnéticas deste material de diagnóstico, com a qual o núcleo do monofásico pólo transformadores de distribuição é fabricado. Tais diagnósticos, estudos complementares para avaliar a vida destas equipas, comparando a qualidade dos materiais que são fabricados utilizando "métodos não convencionais" quando presentes condições de falha, aumentando a experiência em sistemas de distribuição de energia elétrica. O trabalho foi realizado utilizando técnicas de caracterização para a chapa de aço usando (difração de raios-X) ou DRX e MEV no ambiente (microscopia eletrônica de varredura) ou E-SEM permitindo uma visão mais clara dos fenômenos envolvidos o comportamento da chapa de aço silício. Embora realizado anteriormente amostras metalográficas de falha para verificar o tamanho do grão impurezas ou inclusões que pode ter afectado a qualidade do material durante a operação de equipamento de análise, de XRD é aplicada e a equação de Scherrer é usado, verificando o tamanho dos cristais (grãos) em escala nano, e, em seguida, com a fórmula perdas Steinmetz são avaliados devido à histerese magnética também afecta significativamente a operação. Total do núcleo são as perdas devido à presença de óxidos de Tipo D e alterações no tamanho do cristal (grão).

Palavras-chave: Análise de fracasso, chapa de aço silício, transformadores de distribuição, DRX e SEM-E.

\section{Introducción}

En la presente investigación se presentan resultados de una investigación que inició en el año 2006, para evaluar los elementos químicos con las cuales se fabricaba la chapa de acero al silicio que se utiliza en los núcleos de los transformadores de distribución monofásicos de poste. En esa oportunidad, ese estudio era para evaluar la factibilidad técnica y económica de fabricar ese material, que se había convertido en una materia prima de difícil consecución para las pequeñas empresas que fabrican o reparan dichos equipos [1]. Luego de esa primera evaluación, se realizó otro proyecto de investigación que permitió el estudio metalográfico de este material, luego que hubiera presentado fallas de tipo térmicas, por arco eléctrico o descargas parciales [2-3], que para el sector eléctrico representan métodos de análisis no convencionales. Después de esos resultados, fueron aplicadas otras técnicas de caracterización en escala nanométrica cuyos resultados se muestran en la presente investigación, y que sirvieron para evaluar además, cuál era la influencia de las condiciones ambientales en las fallas que presentaban los transformadores de distribución [4].

Para realizar estas pruebas experimentales, fueron recolectadas muestras de equipos fallados procedentes de diferentes regiones de Colombia: Bogotá, Cundinamarca, Antioquía, Caldas y Pacífico, las mismas fueron clasificadas de acuerdo al tipo de falla y causas asociadas. Los cambios que presentaba la microestructura pudieron relacionarse con algunas características físicas, permitiendo determinar las pérdidas totales, siendo ésta una condición de suma importancia para las empresas que fabrican o reparan estos equipos, mejorando el análisis de si es viable o no, reutilizar esta chapa de acero al silicio [5-6].

En ese mismo orden de ideas, en el año 2015; se ha publicado un experimento para esta chapa de acero al silicio [7] que indica que el calentamiento rápido tiene un fuerte efecto sobre el comportamiento de recristalización en los aceros eléctricos no orientados, lo que facilita la optimización de la microestructura y la textura, 
especialmente en la estructura de grano sin perder las propiedades magnéticas [8]. Al realizar estas pruebas a la chapa de acero al silicio se evaluaron los cambios que sufre el material que evidentemente tiene relación con el experimento anterior, porque orientan las investigaciones futuras a realizar tratamientos térmicos para mejorar el tamaño de grano teniendo cuidado con la presencia de óxidos tipo $D$ tanto finos como gruesos, cuya influencia pudiera deberse a un cambio de fase. Los resultados de estas investigaciones complementan de manera importante los resultados obtenidos y mejoran la experticia para el estudio de fallas los métodos no convencionales utilizados en la presente investigación, tal y como se indican a continuación [9-13]:

\section{Materiales utilizados}

En la presente investigación se utilizaron restos de equipos fallados suministrados por la empresa colombiana FYR Ingenieros C.A, que se encarga de repararlos o fabricarlos. La Tabla 1 muestra cuarenta y siete (47) transformadores, de los cuales fueron seleccionados dieciséis (16) de acuerdo al tipo de falla por una parte, y por la otra, por cuestiones de costos para algunas pruebas experimentales, además de uno (01) que sirve de referencia para la muestra patrón. A continuación lo indicado:

Tabla 1. Tipo de falla y departamento de procedencia.

\begin{tabular}{ccc}
\hline $\begin{array}{c}\text { Departamento de Procedencia del } \\
\text { Transformador }\end{array}$ & Tipo de Falla & Cantidad \\
\hline \multirow{3}{*}{ Caldas } & Térmica & 1 \\
& Arco Eléctrico & 1 \\
& Descarga Parcial & 1 \\
& Térmica & 1 \\
\hline \multirow{2}{*}{ Antioquia } & Arco Eléctrico & 2 \\
& Descarga Parcial & 1 \\
& Térmica & 1 \\
\hline \multirow{2}{*}{ Pacífico } & Arco Eléctrico & 1 \\
& Descarga Parcial & 1 \\
\hline \multirow{2}{*}{ Cundinamarca } & Térmica & 1 \\
\hline \multirow{2}{*}{ Bogotá } & Térmica & 1 \\
\hline TOTAL & Arco Eléctrico & 3 \\
\hline & Descarga Parcial & 1 \\
\hline
\end{tabular}

\section{Metodología}

De los resultados obtenidos en el estudio estadístico aplicando el software SPSS (Stactistical Package Social Science) con las muestras de equipos fallados se hace énfasis, en la falla de mayor incidencia que es la debida a arco eléctrico[14], dado que cuando se identifican los modos de falla por condiciones ambientales en los transformadores de distribución, determinando causas reales que originan los mismos, aplicando "métodos no convencionales" a los materiales de fabricación, se deben caracterizar condiciones físicas que estos presenten realizando pruebas E- SEM y XRD a fin de evaluar el estado de la chapa de acero al silicio de los transformadores de distribución, luego de presentar una falla, como una continuación de las investigaciones que se vienen desarrollando en el área, tal y como se indicó en la introducción.

\section{Análisis de la microestructura}

Esta prueba no convencional utiliza las normas ASTM E 112-96 o "Standard Test Methods for Determining Average Grain Size" para determinar tamaño de grano y ASTM E 45 o "Standard Test Methods for Determining Quantity and Inclusions Size", preparando probetas en el equipo, y luego de un proceso de pulido y ataque químico, fueron llevadas al analizador de imágenes evaluando cantidad de inclusiones o impurezas y tamaño de grano. 
Pruebas de microscopia electrónica de barrido Se utilizó para esta prueba un (01) Microscopio Electrónico de Barrido Ambiental (ESEM) de última generación que admite el análisis de muestras en estado natural o bajo condiciones ambientales naturales, sin necesidad de usar técnicas convencionales de preparación de muestras. Este equipo consta de un filamento $\mathrm{W}$ que emite electrones los cuales son enfocados en un haz que bombardea la muestra haciendo que esta emita electrones secundarios electrodispersados y con esta información se adquiere una imagen de morfología y aplicando el sensor de rayos $\mathrm{X}$ se realizan además análisis elementales y mapeos químicos.

\section{Pruebas de difracción por rayos $x$}

Se utilizó para esta prueba un (01) Difractómetro de Rayos X "RIGAKU, MINIFLEX II, operado a temperatura ambiente, equipado con una fuente de radiación de $\mathrm{Cu} K \alpha(\lambda=1,540562 \AA)$, fuente de rayos $X$ a $30 \mathrm{kV}$ y $15 \mathrm{~mA}$, geometría BraggBrentano.

El análisis de fallas en la chapa de acero al silicio incorpora el cálculo de las pérdidas totales en el núcleo de los transformadores de distribución debido a las Corrientes de Foucault e Histéresis Magnética, por lo cual es necesario realizar análisis químico a la chapa de acero al silicio (Ver tabla 4) para determinar el porcentaje de carbono, cuyo referente es utilizado en las propiedades físicas de las aleaciones de acero al silicio entre las cuales se encuentran: resistencia eléctrica, fuerza coercitiva, conductibilidad térmica, inducción remanente y permeabilidad magnética, estas se muestran en la Figura 1.

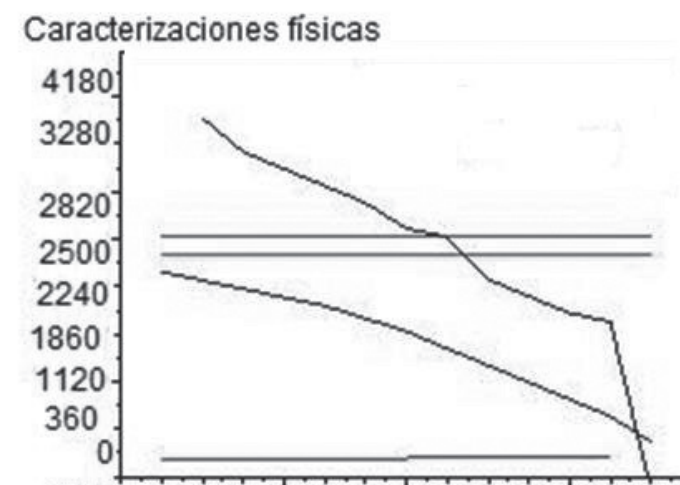

$-0.10 .00 .20 .30 .40 .50 .60 .70 .80 .91 .01 .11 .21 .3$ Porcentaie de carbono de la chapa de acero al silicio

Figura 1. Propiedades físicas de las aleaciones de acero al silicio.
Estas propiedades físicas se determinan aplicando métodos numéricos con el programa Matlab@, obteniendo funciones y gráficos, tal y como se indica a continuación [14-15]:

- Polinomio interpolador de Newton de grado menor o igual que $\mathrm{N}$ que pasa por los puntos $(\mathrm{XK}, \mathrm{YK})=(\mathrm{XK}, \mathrm{f}(\mathrm{XK}))$ para $\mathrm{K}=0,1, \ldots \ldots, \mathrm{N}$ : $P(x)=d 0,0+d 1,1(x-x 0)+d 2,2(x-x o)(x-x 1)+\ldots$ $.+d n, n(x-x o)(x-x 1) \ldots(x-x n-1)$.

- Recta de regresión $y=A x+B$ que mejor se ajusta en el sentido de los mínimos cuadrados a los $\mathrm{N}$ datos $(\mathrm{X} 1, \mathrm{Y} 1), \ldots .,(\mathrm{Xn}, \mathrm{Yn})$.

- Construcción del polinomio de grado $M$ dado por $\operatorname{Pm}(x)=c 1+c 2 x+c 3 x^{2}+\ldots+c m x^{\wedge}(m-$ $1)+c m+1^{\wedge}(x m)$ que mejor se ajusta en el sentido de los mínimos cuadrados a las $\mathrm{N}$ parejas de datos $\{(\mathrm{xk}, \mathrm{yk})\}^{\wedge} \mathrm{N} \mathrm{k}=1$.

Cálculo de pérdidas totales: En un transformador se producen las pérdidas por corrientes de Foucault $(P f)$, pérdidas por histéresis $(P h)$ y en el cobre del bobinado $(P c u)$ [16-17]:

\section{Pérdidas por corrientes de Foucault:}

Se producen en cualquier material conductor cuando se encuentra sometido a una variación de flujo magnético. A continuación en la Tabla 2 se muestran las características para la determinación de las pérdidas de potencia $(\mathrm{W} / \mathrm{kg})$.

Tabla 2. Características de las Pérdidas de Potencia.

\begin{tabular}{cccc}
\hline Espesor & Tolerancia & $\begin{array}{c}\text { Aleación } \\
\text { \% SI }\end{array}$ & $\begin{array}{c}\text { 1 Tesla }(\mathbf{1 0} \\
\text { Gauss) W/kg }\end{array}$ \\
\hline 0,5 & 0,10 & $0,5-1$ & 2,90 \\
0,5 & 0,10 & 2,5 & 2,3 \\
0,35 & 0,10 & 2,5 & 1,7 \\
0,35 & 0,10 & 4 & 1,3 \\
0,35 & 0,10 & 4,5 & 1,2 \\
0,35 & 0,10 & 4,5 & 0,9 \\
\hline
\end{tabular}

Se obtienen a partir de la siguiente expresión, Ecuación 1:

$$
P f=\frac{2,2 f^{2} \beta_{m a ́ x}^{2} \Delta^{2}}{10^{11}}
$$


Dónde:

$P_{f}=$ pérdidas por corriente de Foucault $(\mathrm{W} / \mathrm{kg})$.

$f^{f}=$ frecuencia en $\mathrm{Hz}$.

Bmáx = inducción máxima en Gauss.

$\Delta=$ espesor de la chapa en $\mathrm{mm}$.

\section{Pérdidas por histéresis magnética:}

La histéresis magnética es el fenómeno que se produce cuando la imantación de los materiales ferromagnéticos no solo depende del valor del flujo sino también de los estados magnéticos anteriores, la inducción magnética dependerá del tipo de chapa. Se obtiene aplicando la fórmula de Steinmentz indicada a continuación, Ecuación 2:

$$
P_{h}=K_{f} f \quad B_{m a ́ x}^{n}
$$

Dónde:

$P_{h}=$ pérdidas por Histéresis Magnética (W/kg).

$K_{h}=$ coeficiente de cada material.

$f=$ frecuencia en $\mathrm{Hz}$.

Bmáx = inducción máxima en Tesla.

$n=2$ para $\beta$ >1 Tesla y 1,6 para $\beta<1$ Tesla

\section{Pérdidas totales:}

Las pérdidas de potencia en el hierro $\left(P_{F E}\right)$ o en el núcleo magnético son la suma correspondiente a las pérdidas por Foucault $\left(P_{f}\right)$ y por histéresis $\left(P_{h}\right)$ tal y como, se indica a continuación, Ecuación 3:

Pérdidas Totales en el Núcleo

$$
P_{t}=P_{f}+P_{h}
$$

La ecuación final utilizada Ecuación 4 corresponde a la siguiente expresión [9]:

$$
\mathrm{B}_{\text {máx }}=\sqrt{\left(\frac{10^{11} P_{t}}{10^{11} K_{h} f+2.2 f^{2} \Delta^{2}}\right)}
$$

\section{Resultados}

Pruebas de microscopia electrónica de barrido. Se realizaron pruebas de microscopia electrónica de barrido en ambiente (E-SEM) a todas las muestras de transformadores fallados, sin embargo, a continuación se evalúa el elemento o combinación de estos, que producen precipitados por alta temperatura (inclusiones o impurezas) en la chapa de acero al silicio para la falla por arco eléctrico. Los que se observan en el espectro, son los que afectan la calidad y durabilidad del material, dado que luego de reparados duran menor tiempo en servicio.
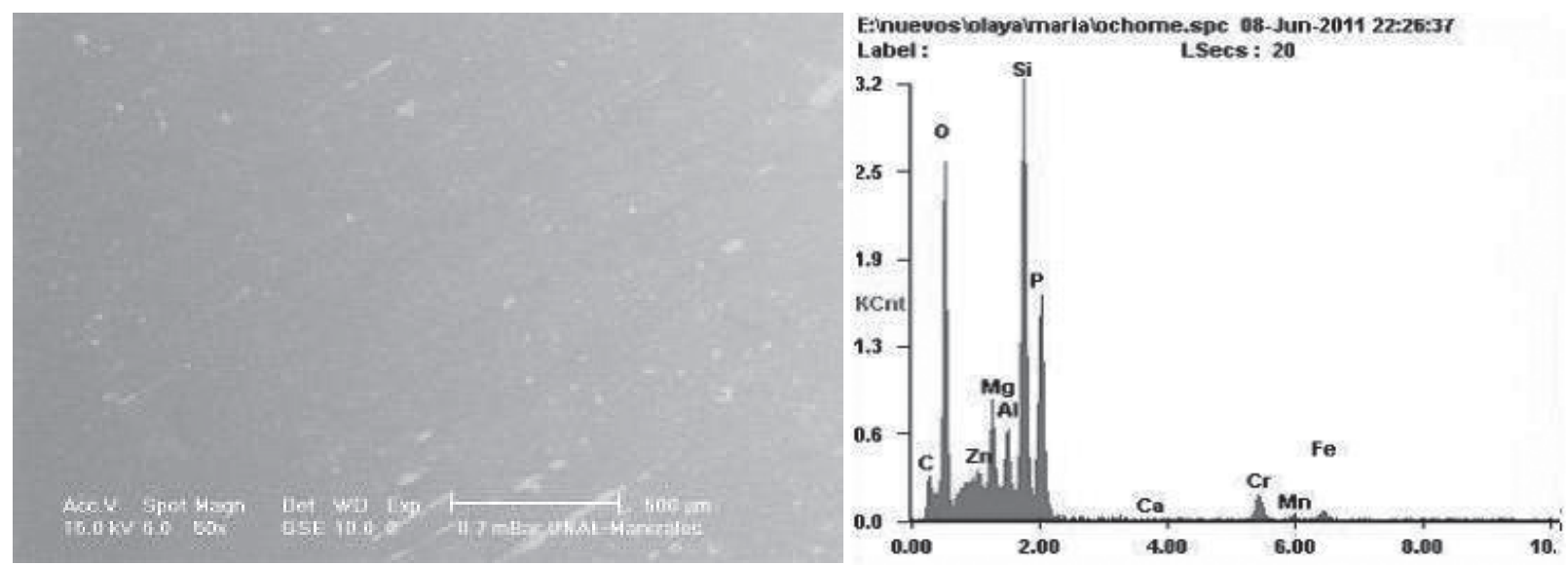

Figura 2. ESEM Muestra Región Antioquia. 
En la Figura 2 de la muestra procedente de la Región Antioquia, el silicio ( $\mathrm{Si}$ ), oxígeno (O) y fósforo $(P)$, son los elementos que presentan
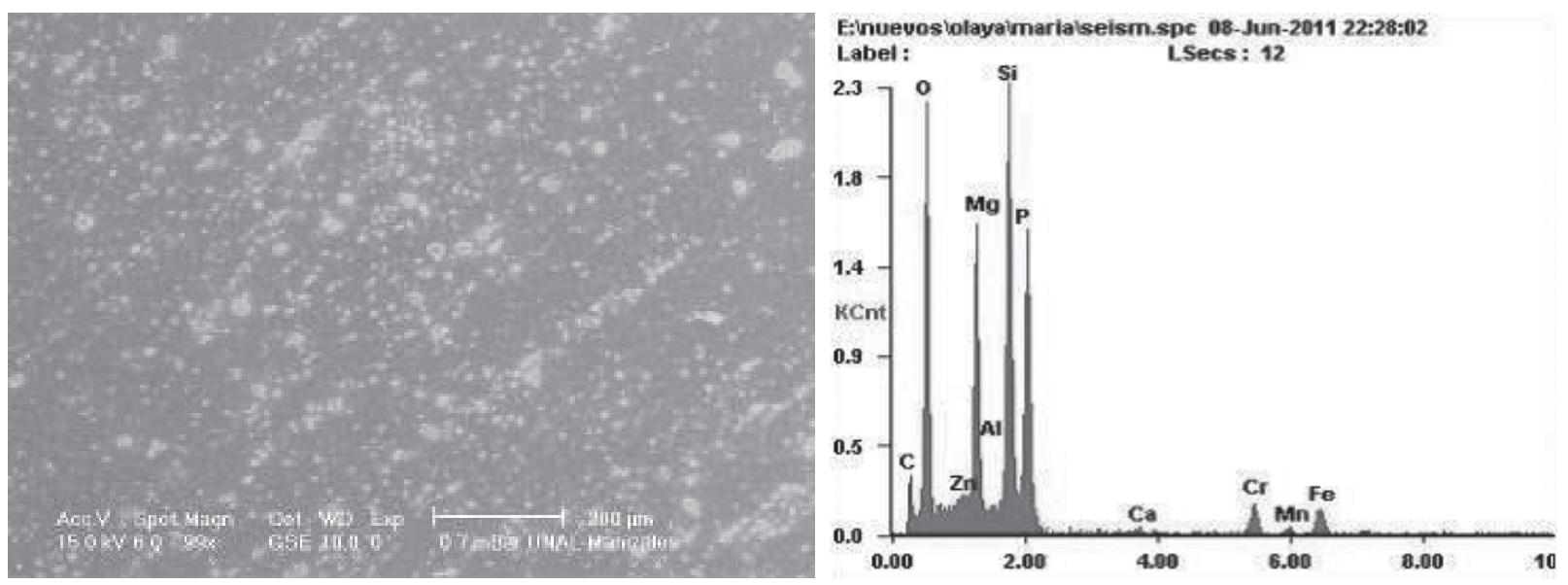

Figura 3. E-SEM Muestra Región Caldas.

En la Figura 3 de la muestra procedente de la Región Caldas, los elementos que producen cambios significativos son el silicio (Si) y el cambios significativos en el espectro e influyen en los precipitados por alta temperatura. oxígeno (O), también el magnesio $(\mathrm{Mg})$ y el fósforo (P) como se indicó anteriormente.
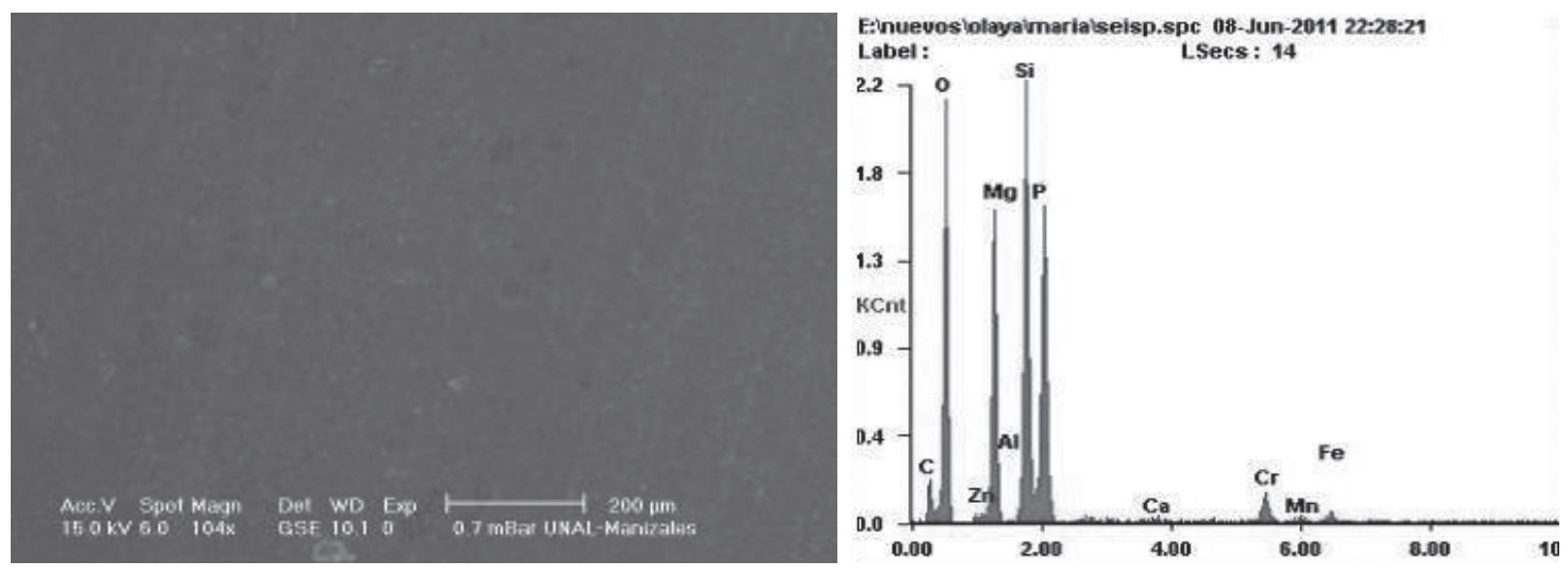

Figura 4. E-SEM Muestra Región Pacífico.

En la Figura 4 cuya muestra procede de la Región Pacífico, hay bastante similitud a la indicada anteriormente de la Región Caldas, lo que pudiera inferir lo siguiente: "a pesar de los cambios de temperatura debido a las distintas ubicaciones geográficas, la respuesta ante la falla se mantiene". 

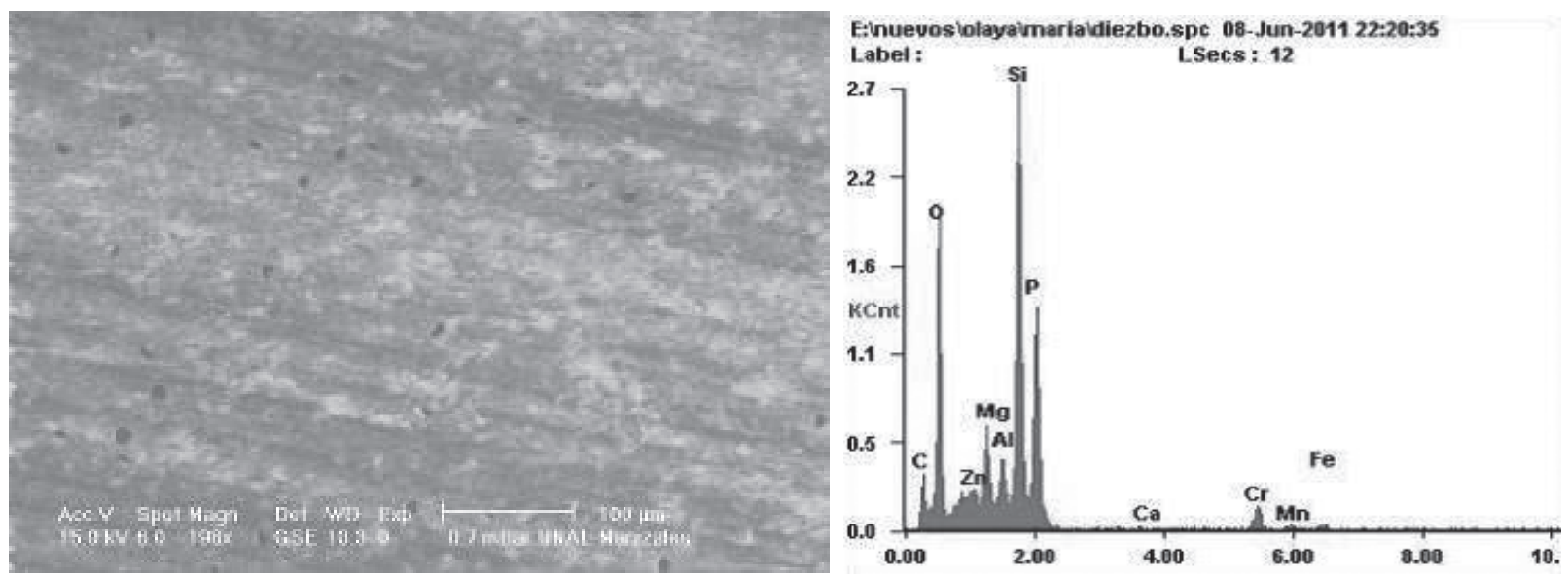

Figura 5. E-SEM Muestra Región Bogotá.

En la Figura 5 de la muestra procedente de la Región Bogotá presenta el mismo comportamiento que la procedente de la región Antioquia, pero es menor la proporción de oxigeno $(\mathrm{O})$ y fósforo $(\mathrm{P})$.
La fotomicrografía no evidencia exceso de óxidos, la muestra está "limpia de precipitados por alta temperatura".
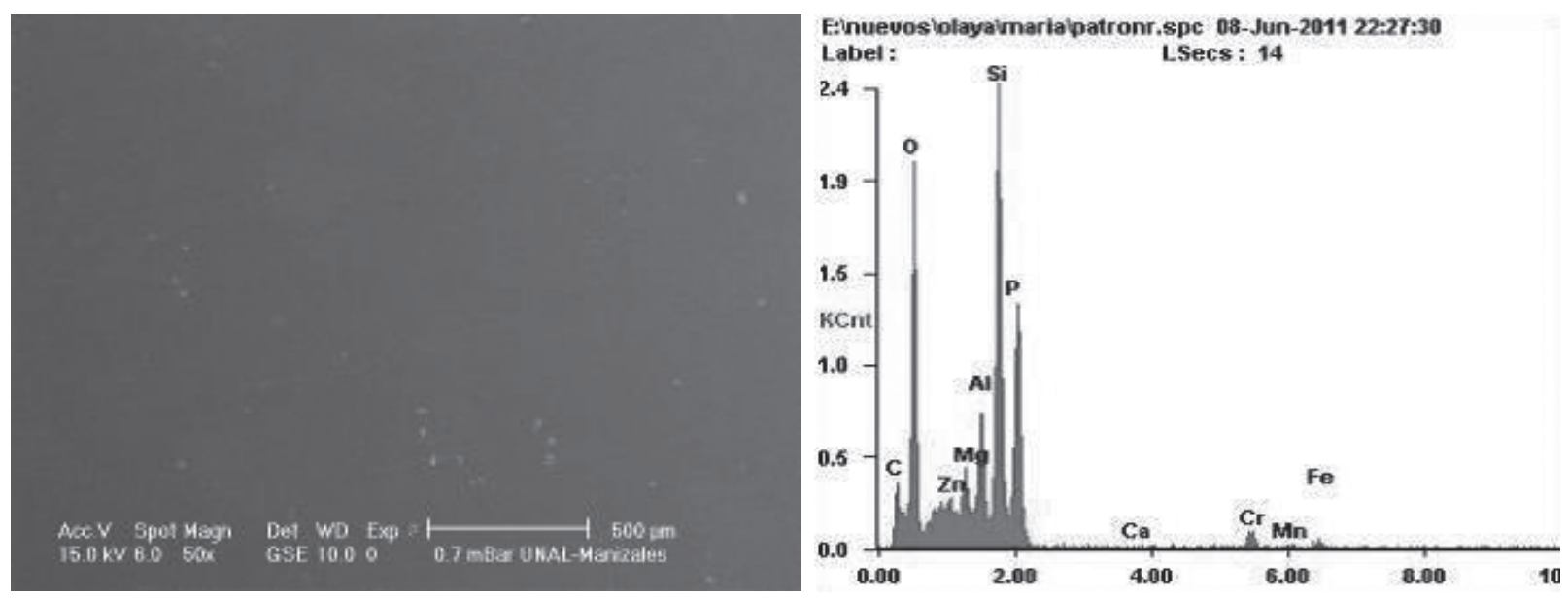

Figura 6. E-SEM Muestra Patrón.

En la Figura 6 correspondiente a la muestra patrón, el silicio (Si) y el oxígeno $(\mathrm{O})$ son los elementos de mayor incidencia, luego el fósforo $(P)$ y aluminio (Al). La fotomicrografía no muestra alteraciones o presencia de "Inclusiones o impurezas".

Los cambios más significados se muestran en la región Caldas y Bogotá, donde la presencia de inclusiones se hace más notable, habría que analizar los cambios de fase que se presentan debido a temperaturas elevadas, tanto en calor como en frío, ya que se presentan alteraciones que deben ser evaluadas en futuras investigaciones. El resto de las muestras procedentes de las otras regiones no presentan cambios significativos.

\section{Pruebas de Difracción por Rayos X.}

A continuación se muestran los resultados obtenidos de acuerdo a la región de procedencia de las muestras de equipos fallados aplicando la Ecuación 5 Debye-Scherrer, se determina el tamaño de grano (crystal size) cuyos resultados se muestran en la Tabla 3. 
rev.ion. 2017;30(2):87-98. Bucaramanga (Colombia).

$$
T=\frac{\mathrm{C} \lambda}{\left(F W H M_{m, S}\right) \cos \theta}=\frac{\mathrm{C} \lambda}{\sqrt{\left(F W H M_{M}^{2}-F W H M_{S}^{2}\right) * \cos \theta}}
$$

Tabla 3. FWHM, 2 theta y Tamaño del cristal.

\begin{tabular}{|c|c|c|c|}
\hline Muestra en estudio & FWHM & 2 theta & Tamaño de grano (cristal) $(\mathrm{nm})$ \\
\hline $\begin{array}{l}\text { Región Antioquia. } \\
\text { Falla Térmica } \\
\text { Región Antioquia. }\end{array}$ & 0,651 & 43,742 & 13,64 \\
\hline $\begin{array}{l}\text { Falla Descarga } \\
\text { Parcial }\end{array}$ & 0,68 & 43,583 & 13,01 \\
\hline $\begin{array}{l}\text { Región Antioquia. } \\
\text { Falla Arco Eléctrico }\end{array}$ & 1,704 & 43,672 & 5,05 \\
\hline $\begin{array}{l}\text { Región Caldas. } \\
\text { Falla Térmica }\end{array}$ & 0,501 & 42,871 & 18,15 \\
\hline $\begin{array}{l}\text { Región Caldas. } \\
\text { Falla Arco Eléctrico }\end{array}$ & 0,144 & 43,505 & \\
\hline $\begin{array}{l}\text { Región Caldas. } \\
\text { Falla Arco Eléctrico/ } \\
\text { Térmica }\end{array}$ & 0,248 & 43,892 & 48,2 \\
\hline $\begin{array}{l}\text { Región Caldas. } \\
\text { Falla Descarga } \\
\text { Parcial }\end{array}$ & 0,248 & 44,09 & 48,23 \\
\hline $\begin{array}{l}\text { Región Pacífico. } \\
\text { Falla Térmica }\end{array}$ & 0,384 & 44,149 & 25,01 \\
\hline $\begin{array}{l}\text { Región Pacífico. } \\
\text { Falla Arco Eléctrico }\end{array}$ & 0,271 & 43,875 & 41,06 \\
\hline $\begin{array}{l}\text { Región Pacífico. } \\
\text { Falla Descarga } \\
\text { Parcial }\end{array}$ & 0,573 & 44,464 & 15,71 \\
\hline $\begin{array}{l}\text { Región } \\
\text { Cundinamarca. } \\
\text { Falla Térmica }\end{array}$ & 0,228 & 43,938 & 57,68 \\
\hline $\begin{array}{l}\text { Región Bogotá. } \\
\text { Falla Arco Eléctrico/ } \\
\text { Térmica }\end{array}$ & 0,296 & 43,318 & 35,59 \\
\hline $\begin{array}{l}\text { Región Bogotá. } \\
\text { Falla Arco Eléctrico }\end{array}$ & 0,234 & 43,888 & 54,36 \\
\hline $\begin{array}{l}\text { Región Bogotá. } \\
\text { Falla Descarga } \\
\text { Parcial }\end{array}$ & 0,553 & 44,045 & 16,31 \\
\hline $\begin{array}{l}\text { Región Bogotá. } \\
\text { Falla Térmica }\end{array}$ & 0,293 & 44,009 & 36,23 \\
\hline Patrón & 0,652 & 43,459 & 13,6 \\
\hline
\end{tabular}

Los resultados obtenidos del análisis químico realizado con el Equipo Spectrovac, Marca Baird. De estos resultados se puede apreciar que hay cambios en el tamaño de grano en escala nanométrica en las regiones de Caldas, Pacífico, Cundinamarca y Bogotá con mayor incidencia debido a fallas térmicas o por arco eléctrico. Los textos de máquinas eléctricas y transformadores establecían que, el tamaño de grano no presentaba cambios, porque este material es magnético blando requiere un rango de temperatura de trabajo superior a $700^{\circ} \mathrm{C}$ para superar el punto de Curie y modificar las propiedades magnéticas, pero, con estos resultados es evidente que el tipo 
de falla y la temperatura ambiente influyen en las condiciones de este material, a pesar de que los valores de temperatura no sobrepasan los $80^{\circ} \mathrm{C}$. El porcentaje de carbono obtenido y comparado con el valor promedio, se muestra en la Tabla 4:

Tabla 4. Análisis Químico.

\begin{tabular}{|c|c|c|}
\hline & Fallas & $\%$ Carbono \\
\hline \multirow{3}{*}{$\begin{array}{l}\text { Región } \\
\text { Antioquia }\end{array}$} & Térmica & 0,193 \\
\hline & Descarga parcial & 0,069 \\
\hline & Arco eléctrico & 0,010 \\
\hline \multirow{4}{*}{ Región caldas } & Térmica & 0,067 \\
\hline & Arco eléctrico & 0,040 \\
\hline & $\begin{array}{l}\text { Arco eléctrico/ } \\
\text { térmica }\end{array}$ & 0,019 \\
\hline & Descarga parcial & 0,014 \\
\hline $\begin{array}{l}\text { Región } \\
\text { Cundinamarca }\end{array}$ & Térmica & 0,031 \\
\hline \multirow{4}{*}{ Región Bogotá } & $\begin{array}{l}\text { Arco eléctrico/ } \\
\text { térmica }\end{array}$ & 0,105 \\
\hline & Arco eléctrico & 0,093 \\
\hline & Descarga parcial & 0,101 \\
\hline & Térmica & 0,1735 \\
\hline \multirow{3}{*}{ Región pacifico } & $\begin{array}{l}\text { Térmica/arco } \\
\text { eléctrico }\end{array}$ & 0,131 \\
\hline & Arco eléctrico & 0,002 \\
\hline & Descarga parcial & 0,033 \\
\hline $\begin{array}{l}\text { Patrón de } \\
\text { referencia }\end{array}$ & & 0,033 \\
\hline
\end{tabular}

En la Tabla 5, se muestra resultados obtenidos, los cuales han sido comparados con las pruebas magnéticas:
En la Tabla 6 se determinan las diferencias del tamaño de grano en nanómetros, luego en la Tabla 7 complementando con la Figura 7 , la relación entre las pérdidas totales: debido al efecto Foucault e histéresis magnética versus tamaño de grano.

Tabla 5. Pérdidas totales.

\begin{tabular}{|c|c|c|c|}
\hline Regiones & $\begin{array}{c}\text { Tipo de } \\
\text { falla }\end{array}$ & $\begin{array}{c}\% \text { de } \\
\text { Carbono }\end{array}$ & $\begin{array}{c}\text { Pruebas } \\
\text { magnéticas }\end{array}$ \\
\hline \multicolumn{4}{|l|}{ Antioquia } \\
\hline Muestra 1 & Térmica & 114,688596 & 85,8702465 \\
\hline Muestra 2 & $\begin{array}{l}\text { Descarga } \\
\text { parcial }\end{array}$ & 117,877042 & 85,8702465 \\
\hline Muestra 3 & $\begin{array}{l}\text { Arco } \\
\text { eléctrico }\end{array}$ & 119,368088 & 85,8702465 \\
\hline \multicolumn{4}{|l|}{ Caldas } \\
\hline Muestra 5 & Térmica & 117,880969 & 85,8702465 \\
\hline Muestra 6 & $\begin{array}{l}\text { Arco } \\
\text { eléctrico }\end{array}$ & 118,586927 & 86,020789 \\
\hline Muestra 7 & $\begin{array}{l}\text { Arco } \\
\text { eléctrico }\end{array}$ & 119,133076 & 85,8702465 \\
\hline Muestra 8 & $\begin{array}{l}\text { Descarga } \\
\text { parcial }\end{array}$ & 119,25154 & 86,173507 \\
\hline \multicolumn{4}{|l|}{ Pacífico } \\
\hline Muestra 9 & Térmica & 158,569707 & 122,932067 \\
\hline Muestra 10 & $\begin{array}{l}\text { Arco } \\
\text { eléctrico }\end{array}$ & 119,555869 & 86,1714773 \\
\hline Muestra 11 & $\begin{array}{l}\text { Descarga } \\
\text { parcial }\end{array}$ & 118,732706 & 86,1735068 \\
\hline \multicolumn{4}{|l|}{ Cundinamarca } \\
\hline Muestra 12 & Térmica & 139,324676 & 103,242098 \\
\hline \multicolumn{4}{|l|}{ Región Bogotá } \\
\hline Muestra 13 & $\begin{array}{l}\text { Arc } \\
\text { eléctrico/ } \\
\text { Térmica }\end{array}$ & 235,265044 & 191,182617 \\
\hline Muestra 14 & $\begin{array}{l}\text { Arc } \\
\text { eléctrico }\end{array}$ & 117,257369 & 86,020789 \\
\hline Muestra 15 & $\begin{array}{l}\text { Arco } \\
\text { eléctrico }\end{array}$ & 118,116709 & 86,020789 \\
\hline Muestra 16 & $\begin{array}{l}\text { Descarga } \\
\text { parcial }\end{array}$ & 116,557365 & 86,018759 \\
\hline Muestra 17 & Térmica & 115,111196 & 86,020789 \\
\hline \multicolumn{4}{|l|}{ Patrón } \\
\hline Muestra 18 & & 131,828066 & 98,5467465 \\
\hline
\end{tabular}

Tabla 6. Tamaño del grano versus el error obtenido.

\begin{tabular}{llllll}
\hline Región & FWHM & THETA & $\begin{array}{c}\text { Tamaño del } \\
\text { Cristal }\end{array}$ & Error & Observaciones \\
\hline Antioquia & 1,704 & 43,672 & 5,05 & 0,3 & $\begin{array}{l}\text { Valor por debajo del } \\
\text { patrón }\end{array}$ \\
Caldas & 0,144 & 43,505 & 0 & 0,00 & $\begin{array}{l}\text { Valor que no puede } \\
\text { apreciarse }\end{array}$ \\
Pacífico & 0,271 & 43,875 & 41,06 & 3,02 & $\begin{array}{l}\text { Valor por encima del } \\
\text { patrón }\end{array}$ \\
Bogotá & 0,234 & 43,888 & 54,36 & 4,00 & $\begin{array}{l}\text { Valor por encima del } \\
\text { patrón }\end{array}$ \\
Patrón & 0,652 & 43,459 & 13,6 & 1 & Valor patrón \\
\hline
\end{tabular}




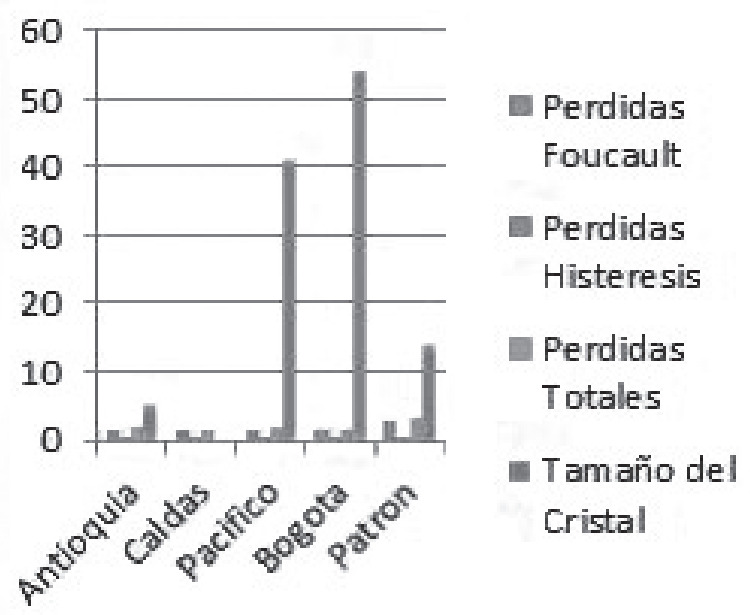

Figura 7. Tamaño de grano \& relación de pérdidas en la falla por arco eléctrico de acuerdo al \% de $\mathrm{C}$.

Todos los tamaños de grano están fuera de los rangos establecidos, es decir, la falla por arco eléctrico "afecta esta condición del material", independiente de la ubicación geográfica del transformador en estudio.

Tabla 7. Pérdidas \& tamaño del cristal de acuerdo al \% de Carbono.

\begin{tabular}{cccc}
\hline Región & $\begin{array}{c}\text { Pérdidas } \\
\text { totales }\end{array}$ & $\begin{array}{c}\text { Tamaño } \\
\text { del Cristal }\end{array}$ & $\begin{array}{c}\text { Error } \\
\text { aproximado }\end{array}$ \\
\hline Antioquia & 1,59157447 & 5,05 & 1,421048634 \\
Caldas & 1,58115902 & 0 & 1,411749125 \\
Pacífico & 1,59407866 & 41,06 & 1,423284518 \\
Bogotá & 1,563432 & 54,36 & 1,395921429 \\
Patrón & 3,16938739 & 13,6 & 2,829810170 \\
\hline
\end{tabular}

\section{Discusión de Resultados}

Pruebas de microscopia electrónica de barrido: Las imágenes muestran los cambios de fase, inclusiones, y otros de la estructura física a fin de compararlas con las fotomicrografías, además de incorporar el espectro con los elementos químicos, que afectan cambios en la aleación e influyen en su "deterioro térmico como evidencia significativa".

\section{Difracción de rayos $\mathrm{x}$ :}

Todos los tamaños de cristal evaluados en la presente investigación procedentes de distintas regiones de Colombia, están fuera de los rangos establecidos, es decir, la falla por arco eléctrico "afecta esta condición del material", independiente de la ubicación geográfica del transformador en estudio. La relación con el tamaño del cristal indicada en el Figura 8 es de suma importancia, porque las muestras procedentes de las Regiones de Bogotá y el Pacífico, presentan un tamaño muy grande en comparación con la muestra patrón, no siendo el caso de las procedentes de las Regiones de Antioquia y Caldas, cuyos tamaños son bastante reducidos, lo cual permite establecer que "la temperatura ambiente influye en los cambios que presenta la microestructura del material".

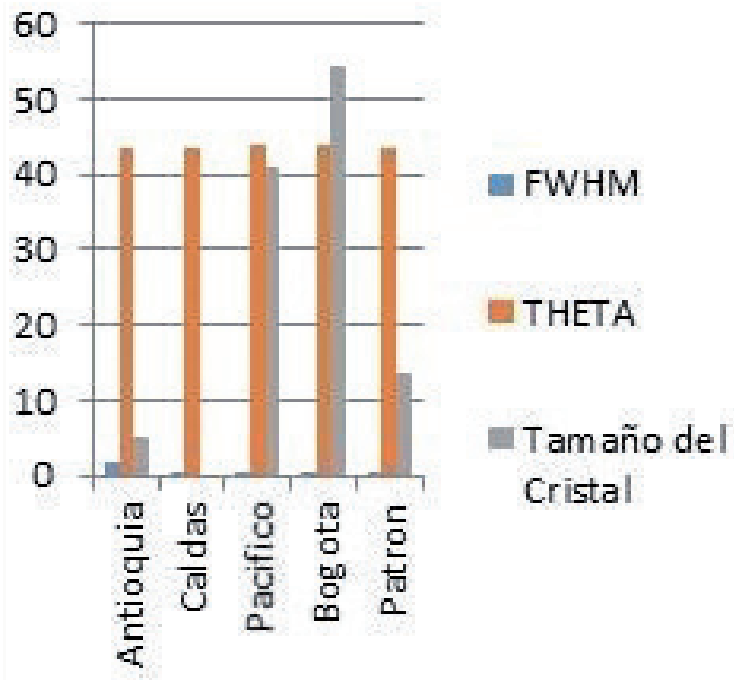

Figura 8. Tamaño de cristal en la falla por arco eléctrico.

Este tipo de material se fabrica utilizando la laminación en frío que para este caso particular debe presentar cero defectos, al igual que el corte de la chapa, sin embargo, debe tenerse en cuenta durante el proceso de fabricación para evitar la aparición de grietas la forma y el tamaño de la muesca alrededor de la punta de la entalla considerando ángulos de alrededor de $45^{\circ}$ y $135^{\circ}$ a la dirección de laminación [16], de lo contrario cuando el material es sometido a fallas se presentan cambios significativos que pueden observarse tanto en las pruebas de E-SEM como $\mathrm{XRD}$ realizadas anteriormente.

Las aproximaciones para obtener las pérdidas totales a partir del porcentaje de carbono de las muestras de equipos fallados traen consigo, errores en la medición, de tal manera que; al calcular las pérdidas totales los resultados obtenidos están por encima de los establecidos por el fabricante, sin embargo, por razones de inmediatez para las 
empresas que reparan o fabrican estos equipos este método resulta bastante útil, siempre y cuando, "se considere dicho error".

Hay una investigación realizada por [18] que utilizaron nano polvos de $\mathrm{SiC}$ con el fin de mejorar las propiedades mecánicas evitando el crecimiento de grano en la zona de agitación (SZ). Esta condición se tomaría como propuesta para comparar las propiedades eléctricas estableciendo mejoras en calidad y costos al material.

\section{Conclusiones}

Hay cambios en la calidad térmica de la chapa de acero al silicio por lo cual no es recomendable su reutilización, se han originados precipitados por alta temperatura (óxidos Tipo $D$ entre 4 y $12 \mu \mathrm{m})$, al igual que disminución del Tamaño de Grano 2 (estándar ASTM o 5.05nm), afectando estas condiciones entre 33,33 y 61,15 +/- 10\% respectivamente.

Las Pérdidas Totales del Núcleo se deben a presencia de óxidos Tipo $D$ y cambios en el tamaño del cristal (grano).

La propiedad física que permite determinar a través de simulación y métodos numéricos las pérdidas totales de la chapa de acero es la saturación magnética, siendo necesario que las investigaciones deben dirigirse hacia la aplicación de estas técnicas de caracterización utilizando modelado numérico y simulaciones, a fin de mejorar el diagnóstico para estos equipos y sistemas eléctricos.

\section{Referencias bibliográficas}

[1] Raffoul Y, Leal I. Determinación de la Factibilidad Técnica y Económica de la Chapa de Acero al Silicio para Transformador Monofásico. Universidad de Carabobo, Facultad de Ingeniería. País: Valencia, Venezuela, 2006. 19. Reed-Hill, Robert. Principios de Metalurgia Física. 6 ed. México: Editorial C.E.C.S.A, 1976.20. Santofimia, M. La Transformaciòn Bainitica Sin Formación de Carburos en Aceros. Universidad Complutense de Madrid, Departamento de Metalurgia Física. País:Madrid, España, 2006.21.Shackelford, J. Ciencia de Materiales para Ingenieros. 4 ed. México: Editorial Prentice Hall, 1998.

[2] Mago M. Análisis de Fallas en Transformadores de Distribución Utilizando Métodos No
Convencionales. Universidad de Carabobo, Área de Estudios de Postgrado. País: Valencia, Venezuela, 2011.

[3] Mago M, Valles L, Olaya J. Evaluación Metalográfica de la Chapa de Acero al Silicio y de los Devanados del Núcleo del Transformador Monofásico Bajo Condición de Falla. Revista INGENIERIA UC. 2011;18(3):51-63.

[4] Mago M. Sistema Estocástico Unificado para la Predicción Confiable de Fallas por Condiciones Ambientales en Transformadores de Distribución. Universidad de Carabobo, Área de Estudios de Postgrado. País: Valencia, Venezuela, 2014.

[5] Norma RA7-60. Valores de Pérdidas Equipos Bajo Condición de Carga. http://www.epm. com.co/site/Portals/0/Users/001/01/1/RA7060.pdf. Consultado: 20-06-12 hora: 10:30

[6] Mago M, Valles L, Olaya J. Análisis de Fallas en Transformadores de Distribución Utilizando el Software Estadístico SPSS (Statistical Package for The Social Sciences). Revista Ingeniería e Investigación Universidad Nacional de Colombia. 2012;32(2):40-5.

[7] Fang F, Xu YB, Zhang YX, Misra RDK, Wang GD. Journal of Magnetism and Magnetic Materials. 2015;381:433-9.

[8] Wang Y, Xu YB, Zhang YX, Liu HT, Wang GD. Journal of Magnetism and Magnetic Materials. 2014;379:161-6.

[9] ASTM D 3906-03. Standard Test Method Determination for Relative X-ray Diffraction Intensities of Faujasite-Type Zeolite Containing Materials.

[10]ASTM D 3942-03. Standard Test Method Determination of the Unit Cell Dimension of a Faujasite-Type Zeolite.

[11] ASTM E-45. Standard Test Methods for DeterminigThe Inclusion Content of Steel.

[12]ASTM E 112-96. Standard Test Methods for Determinig Average Grain Size.

[13] Forero A. Laboratorio de Metales. Universidad Nacional de Colombia. Facultad de Ingeniería. País: Bogotá, Colombia, 1999.

[14] Mathews J, Fink K. Métodos Numéricos con Matlab. 3 ed. Madrid, España: Editorial Prentice Hall, 2015.

[15] Lajtin Y. Metalografía y Tratamiento Térmico de los Metales. 3 ed. Moscú, URSS: Editorial MIR, 1983.

[16]Staff EE. Circuitos Magnéticos y Transformadores. Argentina: Editorial Reverté; 1981. 
[17] Mago M, Vallés L, Olaya J, Zequera M, Vera J. Method for Determining Total Losses in Distribution Transformers from The Percentage of Carbon Silicon Steel Sheet, Medellín, Colombia. Revista DYNA. 2016;83(198):148-53.

[18] Sun Q, Zan D, Chen J, Pan H. Analysis of edge crack behavior of steel sheet in multipass cold rolling based on a shear modified GTN damage model. Theoretical and applied fracture mechanics. 2015;80:259-66.

[19] Tebyani SF, Dehghani K. Effects of SiC nanopowders on the mechanical properties and microstructure of interstitial free steeljoined via friction stir spot welding. Department of Mining and Metallurgical Engineering, Amirkabir University of Technology (Tehran Polytechnic), Tehran, Iran. Materials and Desing. 2016;90:660-8. 